12,18

\title{
Динамика цепочек углеродных нанотрубок, расположенных на плоских подложках
}

\author{
(C) А.В. Савин ${ }^{1,2}$, О.И. Савина ${ }^{2}$ \\ ${ }^{1}$ Федеральный исследовательский центр химической физики им. Н.Н. Семенова РАН (ФИЦ ХФ РАН), \\ Москва, Россия \\ ${ }^{2}$ Российский экономический университет им. Г.В. Плеханова, \\ Москва, Россия \\ E-mail: asavin@center.chph.ras.ru
}

Поступила в Редакцию 3 сентября 2020 г.

В окончательной редакции 3 сентября 2020 г.

Принята к публикации 23 сентября 2020 г.

Исследованы стационарные состояния массива одностенных углеродных нанотрубок лежащего на плоской подложке, образованной поверхностью молекулярного кристалла. Численное моделирование показало, что при слабом взаимодействии с подложкой нанотрубкам энергетически более выгодно образовывать многослойные упаковки, а при сильном (при взаимодействии с поверхностью кристалла $h-\mathrm{BN}$ ) однослойную упаковку (цепочку на поверхности подложки). Проведено моделирование динамики цепочек нанотрубок. Показано, что сверхзвуковые акустические солитоны могут существовать только в цепочках нанотрубок малого диаметра $D<0.8 \mathrm{~nm}$, а движение солитона всегда сопровождается потерей энергии на возбуждение внутренних колебаний нанотрубок. Проведен анализ малоамплитудных колебаний. Показано, что в конечной цепи нанотрубок на плоской подложке локализация колебаний возможна только на концевых нанотрубках или на нанотрубке, образующей структурный дефект цепи.

Ключевые слова: углеродная нанотрубка, плоская подложка, многослойные упаковки, цепочки нанотрубок, акустический солитон, локализованные колебания.

DOI: 10.21883/FTT.2021.01.50412.183

\section{1. Введение}

Углеродные нанотрубки (УНТ) представляют собой макромолекулы цилиндрической формы диаметра от $0.4 \mathrm{~nm}$ и длиной до нескольких $\mu \mathrm{m}$, состоящих из одного или нескольких слоев графена. Впервые похожие структуры были обнаружены при термическом разложении окиси углерода на железном контакте [1]. Сами нанотрубки получены значительно позже как побочные продукты синтеза фуллерена $\mathrm{C}_{60}$ [2]. В настоящее время УНТ привлекают внимание благодаря своим уникальным свойствам [3]. Они могут быть легко синтезированы с наперед заданными геометрическими характеристиками (с требуемыми диаметром, длиной и хиральностью) [4,5], а из них получены пучки параллельных нанотрубок [6,7]. Такие материалы, также называемые лесом или массивом УНТ, обладают еще более высокими механическими свойствами, чем отдельные нанотрубки, благодаря наличию между ними вандер-ваальсовых взаимодействий [8]. Невалентное взаимодействие нанотрубки с подложкой может изменять цилиндрическую форму нанотрубки, делая ее более сплюснутой $[9,10,11]$.

В настоящей работе мы рассмотрим массивы УНТ, расположенные на плоской подложке, образованной поверхностью молекулярного кристалла. С использованием 2D-цепной модели будет показано, что при слабом взаимодействии с подложкой нанотрубкам энергетиче- ски более выгодно образовывать многослойные упаковки, а при сильном (при взаимодействии с поверхностью кристалла $h-\mathrm{BN})$ - однослойную упаковку (цепочку на поверхности подложки). Далее для цепочек УНТ будут получены дисперсионные кривые малоамплитудных колебаний и промоделирована нелинейная динамика. Будет показано, что сверхзвуковые акустические солитоны могут существовать только в цепочках нанотрубок малого диаметра $d<0.8 \mathrm{~nm}$, а движение солитона всегда сопровождается потерей энергии на возбуждение внутренних колебаний нанотрубок. Также будет проведен анализ локализации малоамплитудных колебаний в конечной цепи УНТ.

\section{2. Модель массива УНТ на плоской подложке}

Для описания массива (пучка) нанотрубок удобно использовать двухмерную модель системы молекулярных циклических цепей $[12,13]$. Для одностенной УНТ со структурой зигзаг (с индексом хиральности $(m, 0))$ цепная модель описывает поперечное сечение нанотрубки, образующее кольцевую цепочку из $N=2 m$ эффективных частиц, соответствующих продольным линиям атомов нанотрубки.

Будем считать, что все нанотрубки в массиве ориентированы вдоль оси $z$, тогда одной эффективной 


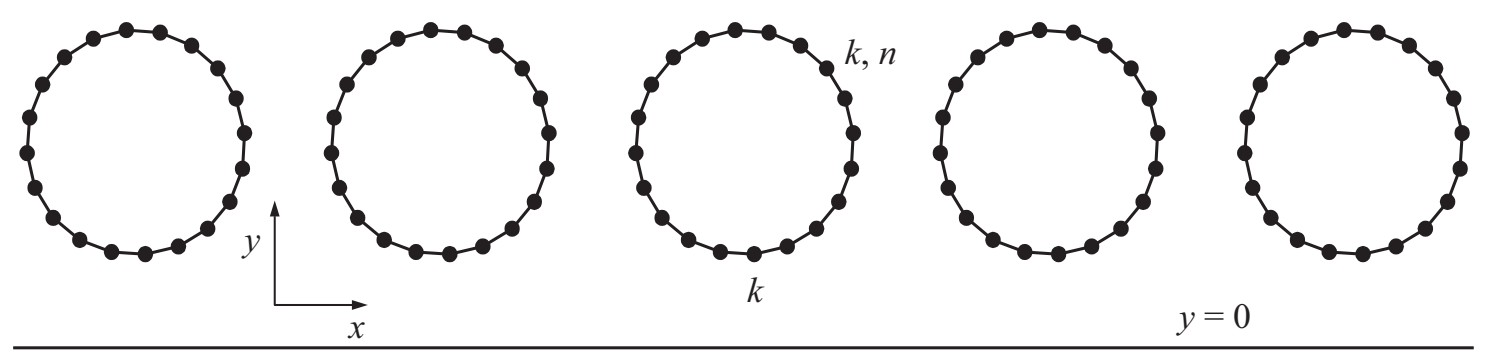

Рис. 1. 2D-модель массива нанотрубок (циклических цепей в плоскости $x y), k-$ номер нанотрубки (цепи), $n-$ номер узла цепи, линия задает поверхность плоской подложки. Показана цепочка нанотрубок $(10,0)$, расположенная на поверхности кристалла $h-\mathrm{BN}$.

частице соответствуют все атомы нанотрубки, имеющие одинаковые координаты $x, y$. Если эти атомы двигаются синхронно, меняя только координаты $x, y$, но не меняя координату $z$, то гамильтониан одной нанотрубки можно записать как гамильтониан циклической цепи

$$
\begin{aligned}
H= & \sum_{n=1}^{N}\left[\frac{1}{2} M\left(\dot{\mathbf{u}}_{n}, \dot{\mathbf{u}}_{n}\right)+V\left(R_{n}\right)+U\left(\theta_{n}\right)\right. \\
& \left.+W_{0}\left(\mathbf{u}_{n}\right)+\frac{1}{2} \sum_{|k-n|>4} W_{1}\left(r_{n, k}\right)\right],
\end{aligned}
$$

где двухмерный вектор $\mathbf{u}_{n}=\left(x_{n}, y_{n}\right)$ задает координаты $n$-ой частицы цепи, $M$ - масса атома углерода.

Потенциал

$$
V(R)=\frac{1}{2} K\left(R-R_{0}\right)^{2}
$$

описывает продольную жесткость цепи, $K-$ жесткость взаимодействия, $R_{0}-$ равновесная длина связи (шаг цепи), $R_{n}=\left|\mathbf{u}_{n+1}-\mathbf{u}_{n}\right|-$ расстояние между соседними узлами $n$ и $n+1$.

Потенциал

$$
U(\theta)=\varepsilon_{2}[1+\cos (\theta)]
$$

описывает изгибную жесткость цепи, $\theta-$ угол между двумя соседними связями, $\cos \left(\theta_{n}\right)=-\left(\mathbf{v}_{n-1}, \mathbf{v}_{\mathbf{n}}\right) / R_{n-1} R_{n}$, вектор $\mathbf{v}_{n}=\mathbf{u}_{n+1}-\mathbf{u}_{n}$.

Параметры потенциалов (2), (3) определены в [12,13] из анализа дисперсионных кривых наноленты графена, продольная жесткость $K=405 \mathrm{~N} / \mathrm{m}$, шаг цепи $R_{0}=r_{c} \sqrt{3} / 2\left(r_{c}=1.418 \AA-\right.$ длина валентной связи $\mathrm{C}-\mathrm{C}$ в листе графена), энергия $\varepsilon_{2}=3.5 \mathrm{eV}$. Диаметр изолированной нанотрубки $(m, 0) \quad D=R_{0} / \sin (\pi / 2 m)$ $\approx 2 m R_{0} / \pi$.

Потенциал $W_{1}\left(r_{n, k}\right)$ описывает слабые невалентные взаимодействия удаленных узлов цепи $n$ и $k$, $r_{n, k}=\left|\mathbf{u}_{k}-\mathbf{u}_{n}\right|-$ расстояние между узлами. Этот потенциал также используется для описания взаимодействий узлов разных цепей (разных нанотрубок). Потенциал невалентного взаимодействия узлов цепей с высокой точностью [14] может быть описан потенциалом Леннарда-Джонса $(5,11)$

$$
W_{1}(r)=\varepsilon_{1}\left[5\left(r_{1} / r\right)^{11}-11\left(r_{1} / r\right)^{5}\right] / 6,
$$

с энергией взаимодействия $\varepsilon_{1}=0.00832 \mathrm{eV}$ и равновесной длиной $r_{1}=3.607 \AA$.

Потенциал $W_{0}$ в гамильтониане цепи (1) описывает взаимодействие узла цепи с подложкой, образованной плоской поверхностью молекулярного кристалла. При моделировании будем считать что поверхность подложки совпадает с плоскостью $y=0$. Для нахождения этого потенциала была численно найдена зависимость энергии взаимодействия атома углерода с подложкой от его расстояния до плоскости поверхности $y$. Вычисления [14] показали, что энергия взаимодействия с подложкой $W_{0}(y)$ с высокой точностью может быть описана $(k, l)$ потенциалом ЛеннардаДжонса

$$
W_{0}(y)=\varepsilon_{0}\left[k\left(h_{o} / y\right)^{l}-l\left(h_{0} / y\right)^{k}\right] /(l-k),
$$

где степень $l>k$. Потенциал (5) имеет минимум $W_{0}\left(h_{0}\right)=-\varepsilon_{0}\left(\varepsilon_{0}-\right.$ энергия связи атома углерода с подложкой, $h_{0}-$ равновесное расстояние от плоскости поверхности подложки).

Для поверхности кристалла льда $I_{h}$ энергия взаимодействия $\varepsilon_{0}=0.029 \mathrm{eV}$, равновесное расстояние $h_{0}=3.005 \AA$, степени $l=10, k=3.5$. Для поверхности кристалла $\alpha$-графита $\varepsilon_{0}=0.052 \mathrm{eV}, h_{0}=3.37 \AA, l=10$, $k=3.75$, для поверхности кристалла гексагонального нитрида бора $(h-\mathrm{BN}) \varepsilon_{0}=0.0903 \mathrm{eV}, h_{0}=3.46 \AA$, $l=10, k=3.75$.

Схематически используемая 2D-модель массива нанотрубок представлена на рис. 1. Здесь нижняя прямая линия $y=0$ показывает положение поверхности подложки, $k$ задает номер нанотрубки (циклической цепи), $n-$ номер узла в цепи.

\section{3. Многослойные упаковки нанотрубок}

На плоской подложке нанотрубки могут образовывать многослойные упаковки, со слоями параллельными поверхности подложки - см. рис. 2. Регулярная $K$-слойная упаковка $(K \geq 1)$ одинаковых нанотрубок задается про- 
Таблица 1. Зависимость нормированной энергии $E / K$ основного состояния многослойной упаковки УНТ от числа слоев $K$ для нанотрубок разного индекса хиральности $(m, 0)$, лежащих на плоской поверхности кристалла льда $I_{h}, h-\mathrm{BN}$ и $\alpha$-графита (значения даны в $\mathrm{eV}$ )

\begin{tabular}{c|c|c|c|c|c|c|c|c}
\hline & $K$ & $(5,0)$ & $(10,0)$ & $(15,0)$ & $(20,0)$ & $(30,0)$ & $(40,0)$ & $(50,0)$ \\
\hline & 1 & 6.3210 & 2.9167 & 1.6813 & 1.0024 & 0.2053 & -0.3515 & -0.9095 \\
\hline & 2 & 6.2621 & 2.8279 & 1.5710 & 0.8734 & 0.0442 & -0.5251 & -1.0813 \\
\hline Лед $I_{h}$ & 3 & 6.2408 & 2.7976 & 1.5337 & 0.8301 & -0.0099 & -0.5859 & -1.1403 \\
\hline & 4 & 6.2301 & 2.7823 & 1.5151 & 0.8084 & -0.0372 & -0.6170 & -1.1703 \\
\hline & 6 & 6.2193 & 2.7670 & 1.4964 & 0.7867 & -0.0642 & -0.6485 & -1.2002 \\
\hline & 1 & 6.0275 & 2.5181 & 1.1921 & 0.4108 & -0.7538 & -1.8062 & -2.8244 \\
\hline & 2 & 6.1081 & 2.6248 & 1.3248 & 0.5797 & -0.4048 & -1.2033 & -1.9828 \\
\hline & 3 & 6.1375 & 2.6618 & 1.3696 & 0.6349 & -0.3030 & -1.0247 & -1.7306 \\
\hline & 4 & 6.1524 & 2.6804 & 1.3922 & 0.6623 & -0.2545 & -0.9407 & -1.5977 \\
\hline & 6 & 6.1673 & 2.6991 & 1.4145 & 0.6894 & -0.2077 & -0.8600 & -1.4783 \\
\hline & 1 & 6.2107 & 2.7681 & 1.5013 & 0.7911 & -0.0916 & -0.7945 & -1.4473 \\
\hline & 2 & 6.2044 & 2.7523 & 1.4802 & 0.7674 & -0.1021 & -0.7411 & -1.3399 \\
\hline & 3 & 6.2022 & 2.7471 & 1.4731 & 0.7594 & -0.1072 & -0.7291 & -1.3126 \\
\hline & 4 & 6.2010 & 2.7444 & 1.4696 & 0.7554 & -0.1100 & -0.7238 & -1.2995 \\
\hline
\end{tabular}

дольным периодом упаковки $a$ и положением $K$ нанотрубок в одной поперечной элементарной ячейке. Найдем основные состояния таких упаковок.

Пусть координаты узлов $k$-ой нанотрубки $(k$-той циклической цепи) с индексом хиральности $(m, 0)$ задаются $2 N$-мерным вектором $\mathbf{x}_{k}=\left(\mathbf{u}_{k, n}\right)_{n=1}^{N}, \quad N=2 m$, $k=1, \ldots, K$. Тогда энергия деформации нанотрубки

$$
P_{1}\left(\mathbf{x}_{k}\right)=\sum_{n=1}^{N}\left[V\left(R_{n}\right)+U\left(\theta_{n}\right)+W_{0}\left(y_{n}\right)+\frac{1}{2} \sum_{|l-n|>4} W_{1}\left(r_{n, l}\right)\right] .
$$

Потенциальная энергия упаковки нанотрубок, приходящаяся на одну элементарную ячейку (на один период)

$$
\begin{aligned}
E= & \sum_{k=1}^{K} P_{1}\left(\mathbf{x}_{k}\right)+\sum_{k_{1}=1}^{K-1} \sum_{k_{2}=k_{1}+1}^{K} P_{2}\left(\mathbf{x}_{k_{1}}, \mathbf{x}_{k_{2}}\right) \\
& +\sum_{k_{1}=1}^{K} \sum_{k_{2}=1}^{K} P_{3}\left(\mathbf{x}_{k_{1}}, \mathbf{x}_{k_{2}}\right)
\end{aligned}
$$

где энергия взаимодействия цепей в элементарной ячейке

$$
P_{2}\left(\mathbf{x}_{k_{1}}, \mathbf{x}_{k_{2}}\right)=\sum_{n_{1}=1}^{N} \sum_{n_{2}=1}^{N} W_{1}\left(r_{k_{1}, n_{1}, k_{2}, n_{2}}\right)
$$

а функция

$$
P_{3}\left(\mathbf{x}_{k_{1}}, \mathbf{x}_{k_{2}}\right)=\sum_{n_{1}=1}^{N} \sum_{n_{2}=1}^{N} W_{1}\left(r_{k_{1}, n_{1}, k_{2}, n_{2}}^{\prime}\right)
$$

задает энергию взаимодействия $k_{1}$-ой цепи со сдвинутой вдоль оси $x$ на расстояние $a k_{2}$-ой цепи, расстояние между узлами цепей

$$
r_{k_{1}, n_{1}, k_{2}, n_{2}}^{\prime}=\left[\left(x_{k_{2}, n_{2}}+a-x_{k_{1}, n_{1}}\right)^{2}+\left(y_{k_{2}, n_{2}}-y_{k_{1}, n_{1}}\right)^{2}\right]^{1 / 2} \text {. }
$$

Для нахождения основного состояния упаковки нужно решить задачу на минимум энергии

$$
E \rightarrow \min :\left\{\mathbf{x}_{k}\right\}_{k=1}^{K}, a
$$

т. е. найти минимум функции (7) от $2 N K+1$ переменных $\left\{\left(x_{k n}, y_{k n}\right)\right\}_{k=1, n=1}^{K, N}, a$.

Задача на минимум (8) решалась численно методом сопряженного градиента. Решение показало, что форма основного состояния зависит от значения энергии взаимодействия с подложкой $\varepsilon_{0}$. При слабом взаимодействии с подложкой, образованной поверхностью кристалла льда $I_{h}\left(\right.$ при $\left.\varepsilon_{0}=0.029 \mathrm{eV}\right)$, энергия, приходящаяся на одну нанотрубку $E_{0}=D / K$ монотонно уменьшается с увеличением числа слоев $K$ независимо от радиуса нанотрубки - см. табл. 1. При сильном взаимодействии с подложкой, образованной поверхностью кристалла 

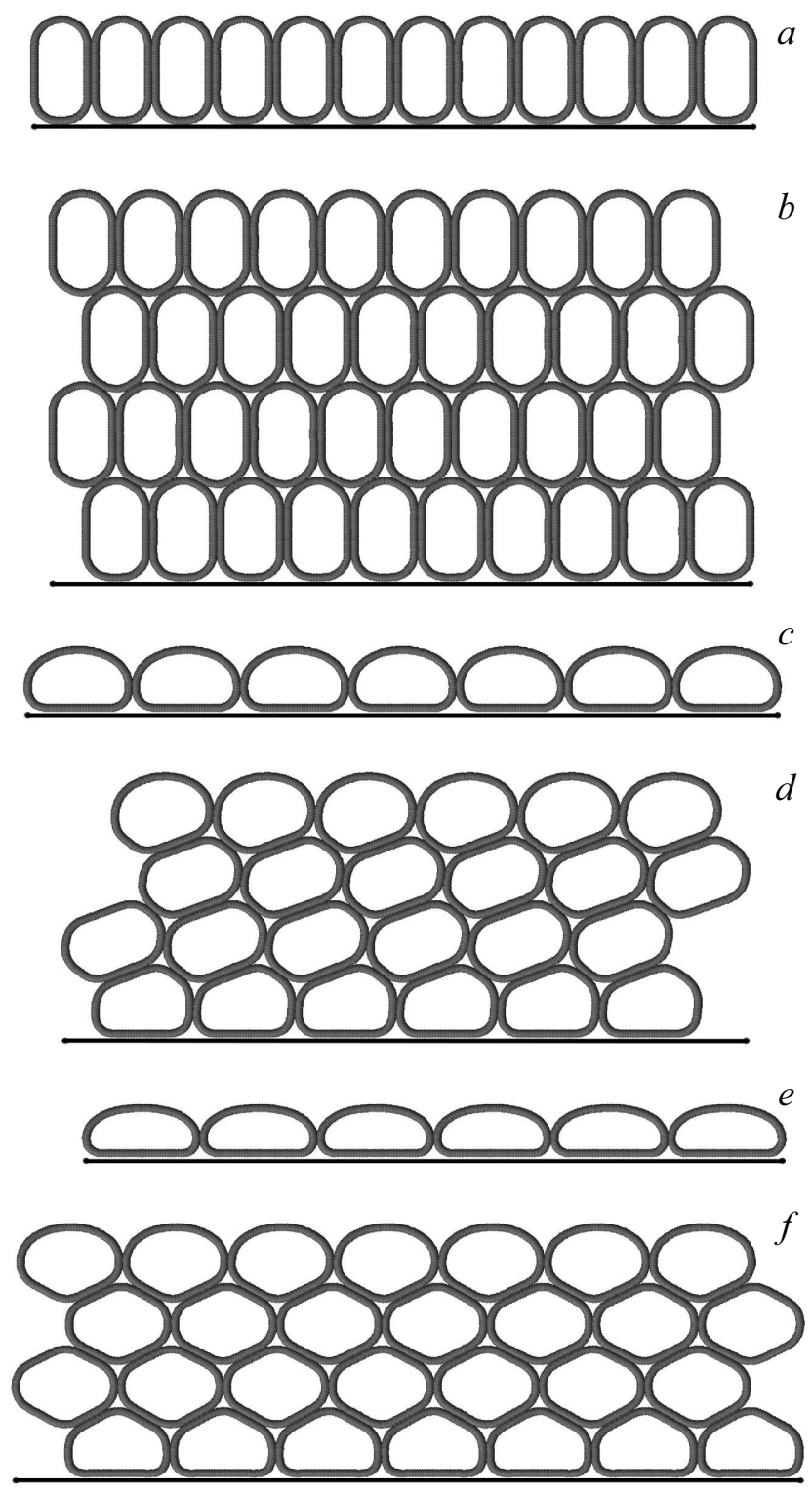

Рис. 2. Вид основного состояния однослойной $(K=1)$ и четырехслойной $(K=4)$ упаковок УНТ с индексом $(50,0)$, расположенных на поверхности кристалла льда $\mathrm{I}_{h}$ (части $a$ и $b$ ), графита $(c$ и $d)$ и $h-\mathrm{BN}(e$ и $f)$.

$h-\mathrm{BN}$ (при $\left.\varepsilon_{0}=0.0903 \mathrm{eV}\right)$, нормированная энергия основного состояния $E_{0}$ монотонно возрастает с увеличением числа слоев $K$ независимо от радиуса нанотрубки. Подложка, образованная поверхностью кристаллического графита, занимает промежуточное положение. Здесь нормированная энергия основного состояния $E_{0}$ слабо зависит от числа слоев упаковки $K$. Энергия монотонно возрастает только для нанотрубок большого диаметра (для нанотрубок с индексом $(40,0)$ и $(50,0))$.

Проведенное моделирование позволяет заключить, что для системы нанотрубок при слабом взаимодействии с подложкой энергетически более выгодно образовывать на поверхности подложки многослойные структуры, а при сильном (при взаимодействии с поверхностью кристалла $h-\mathrm{BN})$ - образовывать однослойную упаковку (цепочку нанотрубок).

Характерный вид упаковок нанотрубок $(50,0)$ для всех типов подложек представлен на рис. 2. При слабом взаимодействии с подложкой нанотрубки большого диаметра сжимаются ортогонально плоскости подложки. Здесь энергетически более выгодно увеличить взаимодействие соседних нанотрубок (увеличить контакт между ними) за счет ослабления их взаимодействия с подложкой. При сильном взаимодействии с подложкой нанотрубки сжимаются параллельно плоскости подложки. Здесь энергетически более выгодно увеличить взаимодействие с подложкой (увеличить контакт с ней) за счет уменьшения взаимодействия соседних нанотрубок (за счет уменьшения контакта между ними).

\section{4. Дисперсионные кривые малоамплитудных колебаний цепи нанотрубок}

На плоской подложке с высокой энергией взаимодействия, такой как поверхность кристалла $h-\mathrm{BN}$, для массива нанотрубок наиболее энергетически выгодной является структура их однослойной упаковки на поверхности подложки. В этом случае нанотрубки образуют цепочку, расположенную вдоль поверхности подложки - см. рис. 1 и 2, $c, e$. Найдем дисперсионные кривые малоамплитудных колебаний такой цепи.

Структура цепочки нанотрубок полностью определяется шагом цепи $a_{0}$ и координатами одной циклической цепи $\mathbf{x}^{0}=\left\{\left(x_{n}^{0}, y_{n}^{0}\right)\right\}_{n=1}^{N}$. В стационарном состоянии узлы $k$-ой нанотрубки в цепи имеют координаты $\mathbf{x}_{k}^{0}=\left\{\left(x_{n}^{0}+k a_{0}, y_{n}^{0}\right)\right\}_{n=1}^{N}$. Для анализа малоамплитудных колебаний удобно перейти к координатам, описывающим смещения звеньев цепей из их положений равновесия $\mathbf{w}_{k}=\mathbf{x}_{k}-\mathbf{x}_{k}^{0}, k=0, \pm 1, \pm 2, \ldots$ Гамильтониан цепи нанотрубок в этих координатах будет иметь вид

$$
H=\sum_{k}\left[\frac{1}{2} M\left(\dot{\mathbf{w}}_{k}, \dot{\mathbf{w}}_{k}\right)+Q\left(\mathbf{w}_{k}, \mathbf{w}_{k+1}\right)\right],
$$

где потенциальная энергия

$$
Q\left(\mathbf{w}_{k}, \mathbf{w}_{k+1}\right)=P_{1}\left(\mathbf{x}_{k}^{0}+\mathbf{w}_{k}\right)+P_{2}\left(\mathbf{x}_{k}^{0}+\mathbf{w}_{k}, \mathbf{x}_{k+1}^{0}+\mathbf{w}_{k+1}\right) .
$$

Гамильтониану цепи (9) соответствует система уравнений движения

$$
-M \ddot{\mathbf{w}}_{k}=Q_{1}\left(\mathbf{w}_{k}, \mathbf{w}_{k+1}\right)+Q_{2}\left(\mathbf{w}_{k-1}, \mathbf{w}_{k}\right),
$$

где функция $Q_{1}\left(\mathbf{w}_{1}, \mathbf{w}_{2}\right)=\partial Q / \partial \mathbf{w}_{i}, i=1,2$. В линейном приближении система уравнений движения (10) принимает форму

$$
-M \ddot{\mathbf{w}}_{k}=B_{1} \mathbf{w}_{k}+B_{2} \mathbf{w}_{k+1}+B_{2}^{*} \mathbf{w}_{k-1},
$$

где матрицы $B_{1}=Q_{11}+Q_{12}, B_{2}=Q_{12}$, а матрицы частных производных

$$
Q_{i j}=\frac{\partial^{2} Q}{\partial \mathbf{w}_{i} \partial \mathbf{w}_{i}}(0,0), \quad i, j=1,2 .
$$




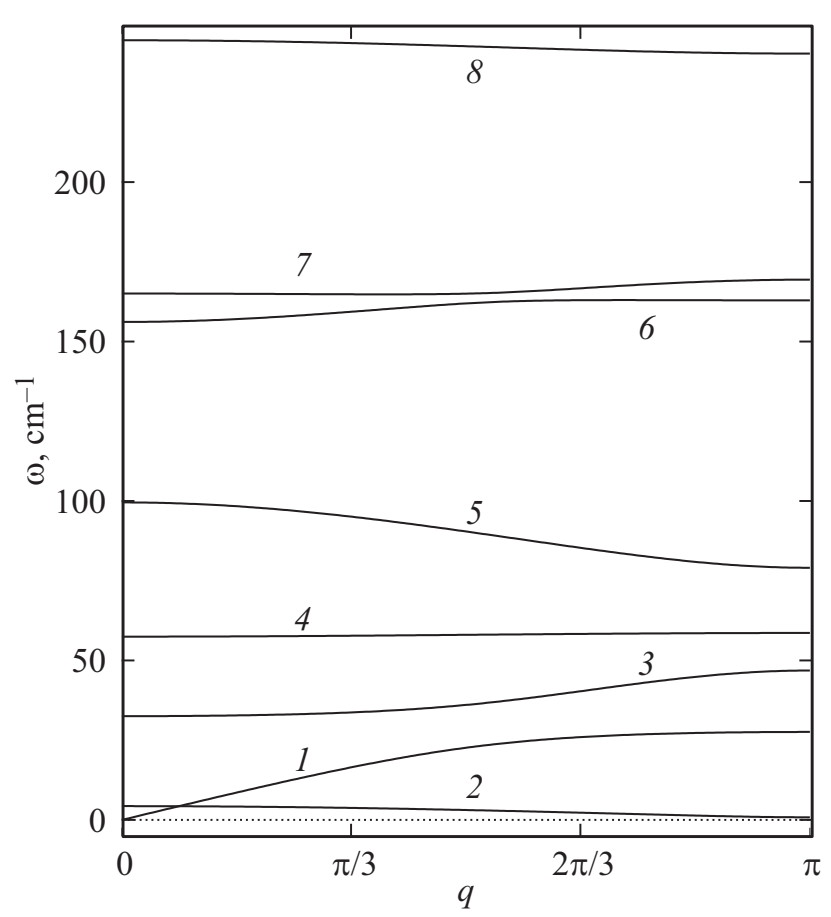

Рис. 3. Вид низкочастотных дисперсионных кривых $\omega_{j}(q)$ цепи нанотрубок $(10,0)$, расположенных на поверхности кристалла $h-\mathrm{BN}$. Номер кривой соответствует номеру дисперсионной кривой.

Решение системы линейных уравнений (11) можно найти в форме волны

$$
\mathbf{w}_{k}(t)=A \mathbf{e} \exp [i(q k-\omega t)]
$$

где $A-$ амплитуда линейной моды, $\mathbf{e}-$ нормированный собственный вектор $(|\mathbf{e}|=1), q \in[0, \pi]-$ безразмерное волновое число, $\omega-$ частота колебаний. Подставив выражение (12) в систему (11), мы получим задачу
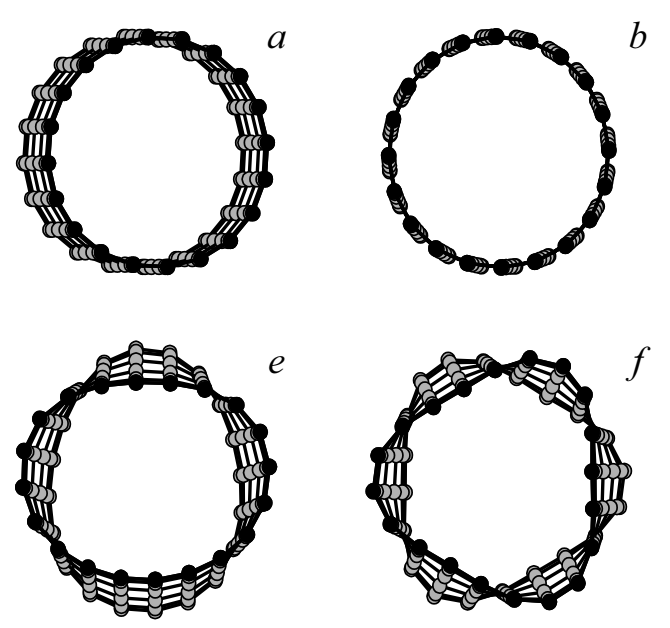

Рис. 4. Вид низкочастотных собственных колебаний цепи нанотрубок $(10,0)$, расположенных на поверхности кристалла $h-\mathrm{BN}$. Показаны колебания с волновым числом $q=0$ с частотами: $a \omega=0 ; b-4.33 ; c-32.53 ; d-57.47 ; e-99.55 ; f-156.14$; $g-165.05 ; h-244.47 \mathrm{~cm}^{-1}$.

на собственные значения эрмитовой матрицы размерности $2 N \times 2 N$

$$
\omega^{2} M \mathbf{e}=\left[B_{1}+B_{2} \exp (i q)+B_{2}^{*} \exp (-i q)\right] \mathbf{e} .
$$

Таким образом, для нахождения дисперсионных кривых $\left\{\omega_{j}(q)\right\}_{j=1}^{2 N}$ нужно для каждого значения волнового числа $0 \leq q \leq \pi$ найти численно все собственные значения задачи (13).

Численное решение задачи (13) показало, что все собственные значения эрмитовой матрицы неотрицательны, что говорит об устойчивости основного состояния цепи нанотрубок на плоской подложке. Отметим, что без подложки линейная цепочка УНТ будет неустойчивой структурой. Все собственные частоты $\left\{\omega_{j}(q)\right\}_{j=1}^{2 N}$ находятся в интервале значений $[0,1500] \mathrm{cm}^{-1}$. Вид низкочастотных дисперсионных кривых $\omega_{j}(q), j=1, \ldots, 8$, для цепи нанотрубок $(10,0)$ (число звеньев цепи $N=20$ ), расположенной на поверхности кристалла $h-\mathrm{BN}$, представлен на рис. 3 , а вид соответствующих собственных колебаний нанотрубки - на рис. 4.

Самая первая дисперсионная кривая $\omega_{1}(q)$ соответствует акустической ветви продольных смещений нанотрубок вдоль поверхности подложки - см. рис. 4, $a$. Данная ветвь выходит из нулевой точки: $\omega_{1}(0)=0$. Скорость длинноволновой акустической моды (скорость звука)

$$
v_{0}=a_{0} \lim _{q \rightarrow 0} \frac{\omega_{1}(q)}{q} .
$$

Значения периода цепи $a_{0}$ и скорости звука $v_{0}$ для цепи нанотрубок разного диаметра представлены в табл. 2. Как видно из таблицы, увеличение диаметра нанотрубки вначале приводит к уменьшению скорости звука в цепи более чем в два раза, а затем к его незначительному росту.

Вторая дисперсионная кривая $\omega_{2}(q)$ описывает крутильные движения нанотрубок, когда они поворачи-
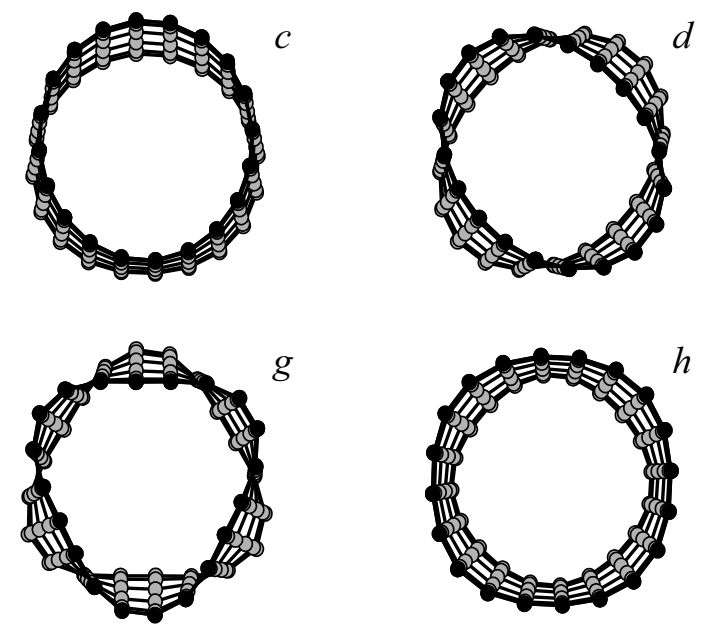
Таблица 2. Зависимость периода $a_{0}$ и скорости звука $v_{0}$ в цепи нанотрубок, лежащей на плоской поверхности кристалла $h-\mathrm{BN}$, от индекса нанотрубки $(m, 0)$

\begin{tabular}{c|c|c|c|c|c|c|c}
\hline$(m, 0)$ & $(5,0)$ & $(10,0)$ & $(15,0)$ & $(20,0)$ & $(30,0)$ & $(40,0)$ & $(50,0)$ \\
\hline$a_{0}$ & 7.052 & 11.025 & 15.086 & 19.512 & 30.293 & 41.844 & $53.829 \AA$ \\
\hline$v_{0}$ & 3879 & 3434 & 2523 & 1866 & 1653 & 1706 & $1840 \mathrm{~m} / \mathrm{s}$
\end{tabular}

ваются как твердое тело вокруг своих центров см. рис. 4, $b$. Третья кривая описывает вертикальные смещения нанотрубок - см. рис. 4,c. Кривые 4 и 5 описывают колебания сжатия поперечного профиля нанотрубок (овальные деформации профиля, рис. 4, $d, e$, кривые 6 и 7 - треугольные деформации профиля $(f, g)$, а кривая $8-$ радиальное деформации профиля $(h$, периодическое изменение диаметра).

\section{5. Продольный ангармонизм цепи нанотрубок}

Если нанотрубку считать абсолютно жесткой круговой цепью (диском), то взаимодействие нанотрубок будет описываться только ангармоническими потенциалами взаимодействия узлов цепей $W_{1}(r)$. В этом случае нанотрубки будут взаимодействовать как большие частицы Леннарда-Джонса. В цепи таких частиц существуют сверхзвуковые акустические солитоны сжатия. Рассмотрим, как будет меняться продольный ангармонизм цепи нанотрубок при учете изменения их формы.

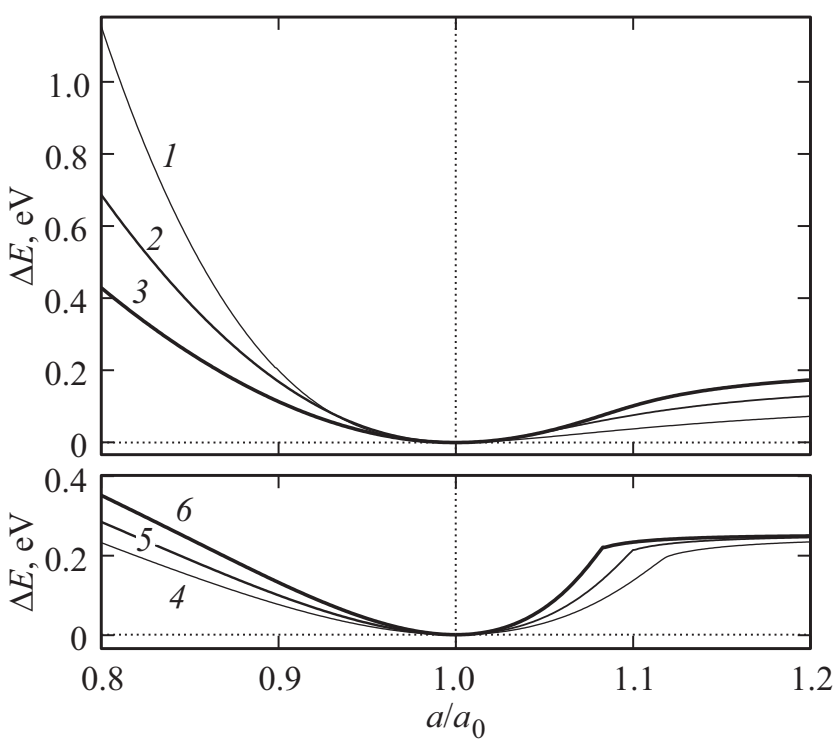

Pис. 5. Зависимость изменения энергии основного состояния $\Delta E$ от изменения шага $a$ для цепи нанотрубок $(m, 0)$, расположенной на поверхности кристалла $h-\mathrm{BN}\left(a_{0}-\right.$ шаг основного состояния цепи). Показаны зависимости для нанотрубок с индексами $(5,0),(10,0),(15,0),(30,0),(40,0),(50,0)-$ кривые $1,2,3,4,5,6$.
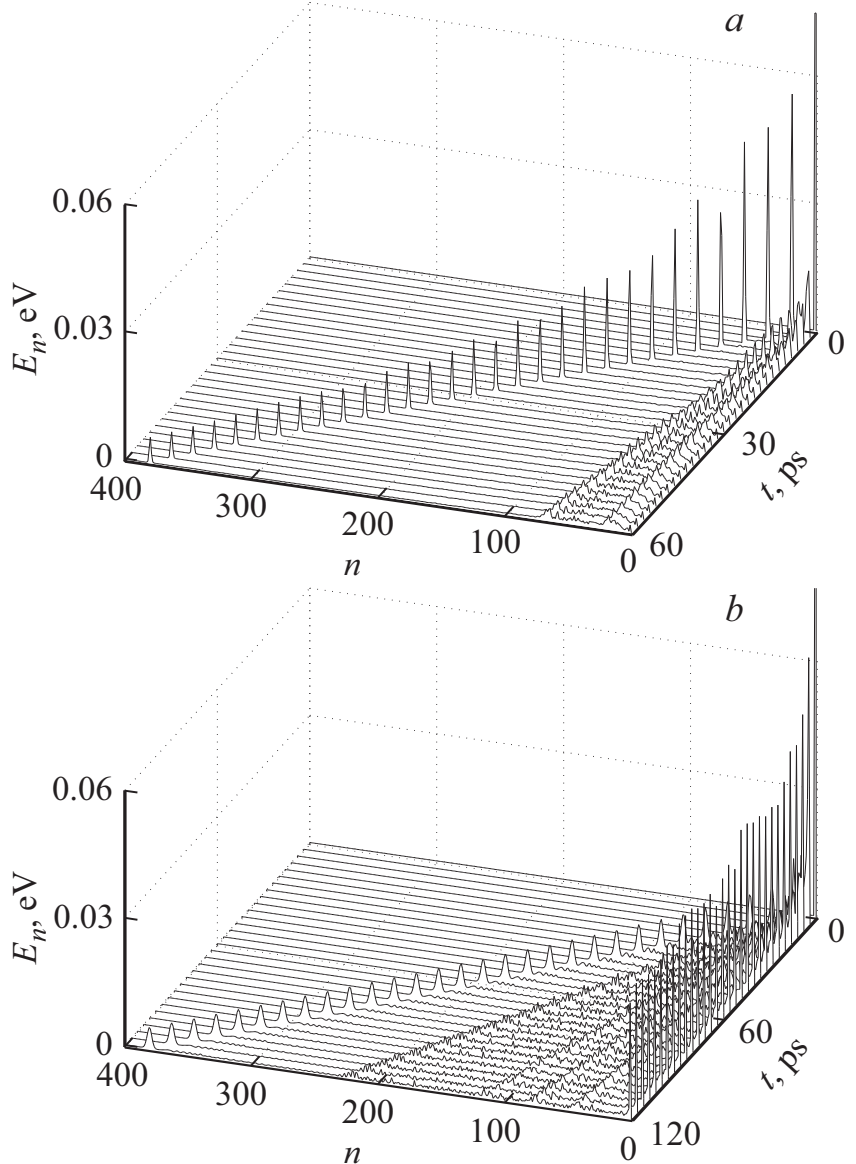

Рис. 6. Изменение распределения энергии в цепи из $K=400$ нанотрубок $(5,0)$ и $(10,0)$ (части $a$ и $b$ ) при начальном уменьшении расстояния между первыми двумя нанотрубками на $\Delta x=0.5 \AA$. При моделировании использовано условие закрепленных краев.

Для нахождения потенциала продольного сжатия цепи нанотрубок решим задачу на минимум энергии основного состояния цепи (8) при различных фиксированных значениях шага цепи $a$. Численно найдем зависимость энергии однородного состояния $E(a)$ от шага цепи. Пусть $a_{0}$ - шаг основного состояния, тогда относительное изменение энергии цепи $\Delta E=E(a)-E\left(a_{0}\right)$. Зависимость $\Delta E$ от относительного изменения шага цепи $a / a_{0}$ представлена на рис. 5 . Как видно из рисунка, для нанотрубок малого диаметра $(5,0)$ и $(10,0)$ цепочка нанотрубок демонстрирует ярко выраженный жесткий 


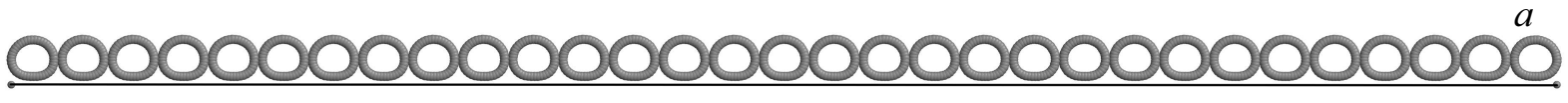

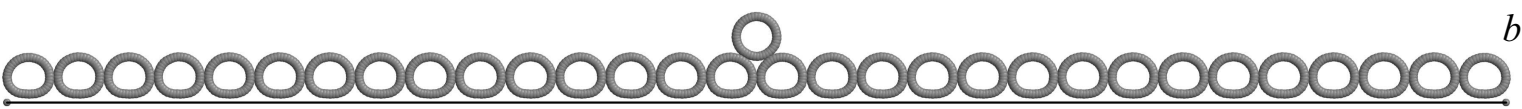

000000000000000000000000000000

$0000000000000000000000000000000000^{\circ}$

\section{0}

Рис. 7. Стационарные состояния цепей из $K=31$ нанотрубок $(20,0)$ на плоской поверхности кристалла $h-\mathrm{BN}$. Часть $a$ показывает основное состояние идеальной цепи, часть $b$ - стационарное состояние цепи со структурным дефектом (одна нанотрубка находится сверху цепи), часть $c$ - состояние цепи с одной нанотрубкой меньшего диаметра (в центре цепи находится нанотрубка с индексом $(15,0))$, часть $d-$ состояние цепи с одной нанотрубкой большего диаметра $(30,0)$, часть $e-$ состояние цепи с одной двустенной нанотрубкой $(11,0) @(20,0)$.

ангармонизм на сжатие и мягкий на растяжение. Для нанотрубок большого диаметра наоборот имеет место мягкий ангармонизм на сжатие и жесткий на растяжение цепи, что объясняется сильным расплющиванием нанотрубок большого диаметра на подложке см. рис. 2, e.

С целью проверки возможности существования в цепи сверхзвуковых акустических солитонов проведем моделирование динамики конечной цепи при начальном изменении расстояния между двумя крайними нанотрубками.

Гамильтониан конечной цепи из $K$ нанотрубок имеет вид

$$
H=\sum_{k=1}^{K}\left[\frac{1}{2} M\left(\dot{\mathbf{x}}_{k}, \dot{\mathbf{x}}_{k}\right)+P_{1}\left(\mathbf{x}_{k}\right)\right]+\sum_{k=1}^{K-1} P_{2}\left(\mathbf{x}_{k}, \mathbf{x}_{k+1}\right),
$$

где $2 N$ мерный вектор $\mathbf{x}_{k}=\left\{\left(x_{k, n}, y_{k, n}\right)\right\}_{n=1}^{N}$ задает координаты звеньев $k$-той циклической цепи. Гамильтониану (14) соответствует система уравнений движения

$$
M \ddot{\mathbf{x}}_{k}=-\partial H / \partial \mathbf{x}_{k}, \quad k=1, \ldots, K .
$$

Возьмем начальное условие, соответствующее основному состоянию цепи. Сдвинем первую нанотрубку так, чтобы расстояние до второй изменилось на $\Delta x$. Далее зафиксируем положение звеньев краевых нанотрубок (кольцевых цепей $k=1, K$ ) и численного проинтегрируем системы уравнений движений (15) с $k=2, \ldots, K-1, K=400$.

Численное интегрирование системы уравнений движения (15) показало, что в цепи нанотрубок самого малого диаметра $(5,0)$ сжатие левого края приводит к образованию сверхзвукового солитона сжатия - см. рис. 6,a. Но движение солитона сопровождается постоянным торможением, вызванным потерями энергии на возбуждение внутренних колебаний нанотрубок. В нанотрубке $(10,0)$ сжатие края приводит к образованию области сжатия, которая двигается практически со скоростью звука - см. рис. 6, $b$. Сжатие левого края для нанотрубок большего диаметра не приводит к образованию солитоноподобных возбуждений. Моделирование динамики цепи нанотрубок $(40,0),(50,0)$ показало, что растяжение левого края тоже не приводит к образованию сверхзвуковых солитонов растяжения. Таким образом, сверхзвуковые акустические солитоны могут существовать только в цепях нанотрубок малого диаметра. Такие солитоны имеют конечную длину пробега, их движение сопровождается потерями энергии на возбуждение у нанотрубок собственных колебаний.

\section{6. Локализация колебаний в цепи нанотрубок}

Для анализа локализации собственных колебаний в цепи нанотрубок рассмотрим конечную цепочку из $K=31$ нанотрубок $(20,0)$ - см. рис. 7. Для нахождения основного состояния цепи $\left\{\mathbf{x}_{k}^{0}\right\}_{k=1}^{K}$ нужно численно решить задачу на минимум энергии системы

$$
E=\sum_{k=1}^{K} P_{1}(\mathbf{x})+\sum_{k_{1}=1}^{K-1} \sum_{k_{2}=k_{1}+1}^{K} P_{2}\left(\mathbf{x}_{k_{1}}, \mathbf{x}_{k_{2}}\right) \rightarrow \min .
$$

Для изучения малоамплитудных колебаний представим смещения узлов циклических цепей в виде $\mathbf{x}_{k}(t)=\mathbf{x}_{k}^{0}+\mathbf{w}_{k}(t)$, где $\left|\mathbf{w}_{k}\right| \ll r_{c}$. Тогда систему уравнений движения цепи нанотрубок можно представить в 


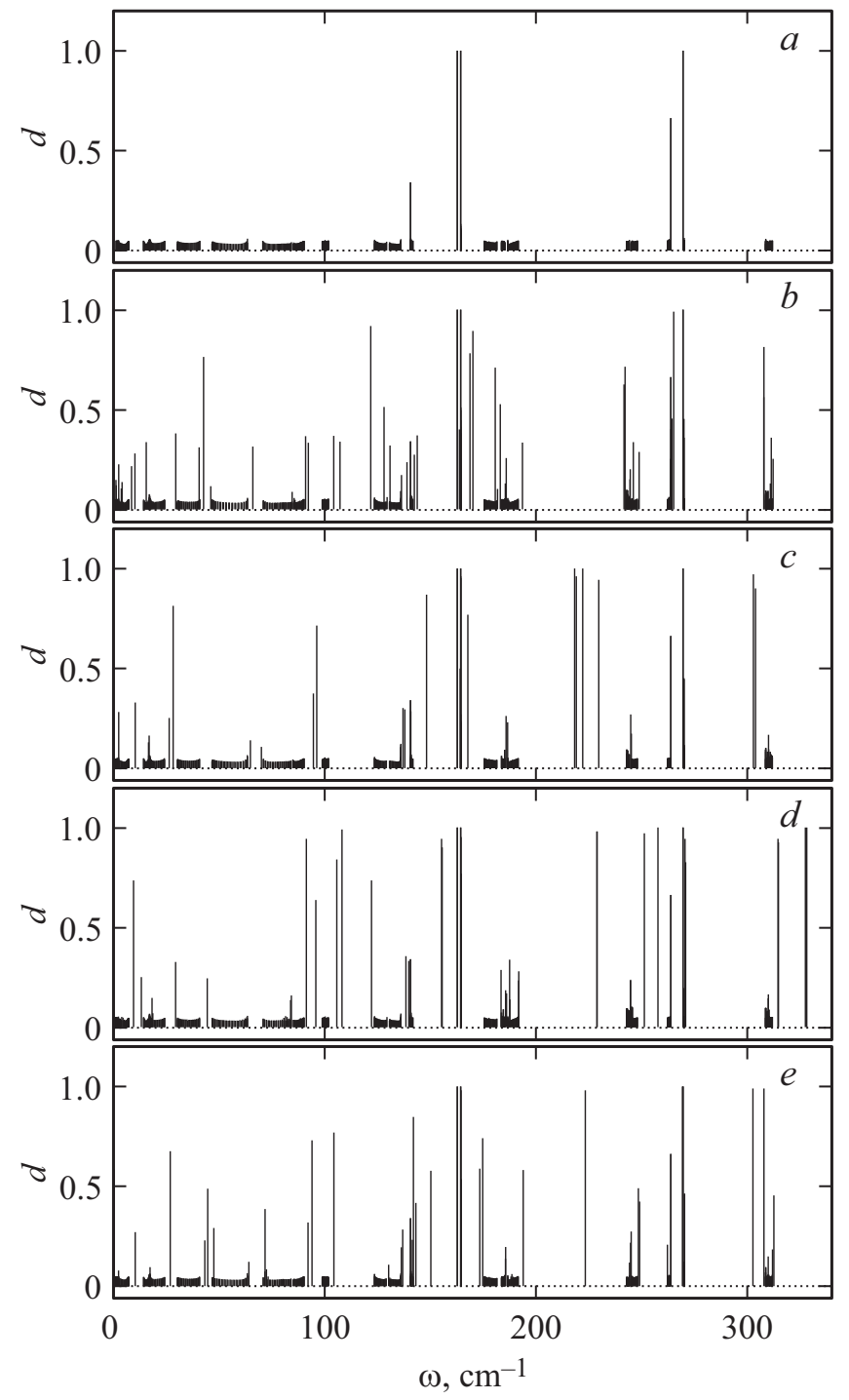

Рис. 8. Зависимость параметра локализации $d$ от частоты колебания $\omega$ для стационарного состояния цепи из $K=31$ нанотрубок $(20,0)$ на плоской поверхности кристалла $h-\mathrm{BN}$. Часть $a$ - для идеальной цепи, часть $b$ - для цепи со структурным дефектом, часть $c$ - для цепи с одной нанотрубкой меньшего диаметра, часть $d-$ для цепи с одной нанотрубкой большего диаметра, часть $e-$ для цепи с одной двустенной нанотрубкой.

виде системы $2 K N$ линейных уравнений с $2 K N$ переменными

$$
-M \ddot{\mathbf{w}}_{k}=\frac{\partial E}{\partial \mathbf{w}_{k}}=\sum_{j=1}^{K} B_{j k} \mathbf{w}_{j}, \quad B_{j k}=\left.\frac{\partial^{2} E}{\partial \mathbf{w}_{k} \partial \mathbf{w}_{j}}\right|_{\left\{\mathbf{x}_{k}^{0}\right\}_{k=1}^{K}} .
$$

Для нахождения всех линейных мод цепи нанотрубок численно были найдены все $2 K N$ собственных вектора и соответствующие собственные значения действительной симметрической матрицы $\mathbf{B}=\left(B_{j k}\right)_{j, k=1}^{K}$. Пусть $\lambda$ и $\mathbf{e}=\left\{\mathbf{w}_{k}^{0}\right\}_{k=1}^{K}$ собственное значение и соответствующий нормированный к единице собственный вектор

$$
\mathbf{B e}=\lambda \mathbf{e}, \quad(\mathbf{e}, \mathbf{e})=\sum_{k=1}^{K}\left(\mathbf{w}_{k}^{0}, \mathbf{w}_{k}^{0}\right)=1
$$

Тогда решение системы уравнений (17) будет иметь вид $\mathbf{w}_{k}(t)=A \mathbf{w}_{k}^{0} \exp (i \omega t), \quad$ где $A-$ амплитуда, а $\omega=\sqrt{\lambda / m}-$ частота линейной моды.

Степень локализации колебания по нанотрубкам удобно характеризовать параметром локализации

$$
d=\sum_{k=1}^{K}\left(\mathbf{w}_{k}^{0}, \mathbf{w}_{k}^{0}\right)^{2}
$$

Для колебания, распределенного по всем нанотрубкам, параметр $d \approx 1 / K$, для колебания, локализованного на одной нанотрубке, $d=1$.

Зависимость параметра локализации $d$ от частоты колебаний цепи нанотрубок $\omega$ представлена на рис. 8 . Как видно из рисунка, для идеальной цепи нанотрубок в области низких частот $\omega<300 \mathrm{~cm}^{-1}$ есть только пять локализованных колебаний (пять частот $\omega_{j}$ с локализацией $\left.d\left(\omega_{j}\right)>1 / 3\right)$. Здесь локализация колебаний происходит только на краевых нанотрубках с номерами $k=1, K$. Число локализованных колебаний резко увеличивается, если в цепи имеются дефекты.

Структурный дефект цепи, когда одна нанотрубка находится сверху цепи, показан на рис. 7, $b$. Стационарное состояние такого дефекта выше по энергии основного состояния цепи на $\Delta E=0.566 \mathrm{eV}$. Наличие дефекта приводит к появлению большого числа собственных колебаний, локализованных на нанотрубке, образующей дефект - см. рис. $8, b$. Наличие в цепи одной отличающейся нанотрубки (одной нанотрубки меньшего, большего диаметра, двустенной нанотрубки - см. рис. $7, c, d, e)$ тоже приводит к появлению колебаний, локализованных на этой нанотрубке, - см. рис. $8, c, d, e$. Моделирование показало, что в цепи большинство собственных колебаний нанотрубки, образующей дефект, остаются локализованными на ней. Поэтому следует ожидать, что такие нанотрубки в массиве нанотрубок будут являться локализованными волноводами.

\section{7. Заключение}

Проведенное численное моделирование показывает, что нанотрубкам при слабом взаимодействии с плоской подложкой энергетически более выгодно образовывать на поверхности подложки многослойные упаковки, а при сильном (при взаимодействии с поверхностью кристалла $h-\mathrm{BN}$ ) - однослойную упаковку (цепочку нанотрубок). При слабом взаимодействии нанотрубки большого диаметра сжимаются ортогонально плоскости подложки, увеличивая контакт между собой, при сильном взаимодействии - сжимаются параллельно подложке, увеличивая контакт с ней. Анализ стационарных состояний цепочек нанотрубок показал, что при 
малых диаметрах нанотрубок их цепочка при сжатии демонстрирует ярко выраженный жесткий ангармонизм, а при больших диаметрах - жесткий ангармонизм при растяжении цепи. Моделирование динамики показало, что сверхзвуковые акустические солитоны могут существовать только в цепочках нанотрубок малого диаметра $D<0.8 \mathrm{~nm}$. Солитон имеет конечную длину пробега, так как его движение всегда сопровождается торможением, вызванным потерями энергии на возбуждение внутренних колебаний нанотрубок.

Проведен анализ малоамплитудных колебаний нанотрубок. Показано, что в конечной цепи нанотрубок локализация собственного колебания возможна только на концевых нанотрубках. Число локализованных колебаний резко увеличивается, если в цепи имеются дефекты (одна нанотрубка находится сверху цепи, в цепи одинаковых одностенных нанотрубок присутствует одна нанотрубка другого диаметра или одна двустенная нанотрубка). В этом случае большинство собственных колебаний нанотрубки, образующей дефект цепи, остаются локализованными на ней.

\section{Финансирование работы}

Исследование выполнено при финансовой поддержке РФФИ и ДНТ в рамках научного проекта № 19-58-45036. Вычислительные ресурсы предоставлены межведомственным суперкомпьютерным центром PAH.

\section{Конфликт интересов}

Авторы заявляют об отсутствии конфликта интересов.

\section{Список литературы}

[1] Л.В. Радушкевич, В.М. Лукьянович. ЖФХ 26, 1, 88 (1952).

[2] S. Iijima. Nature 354, 56 (1991).

[3] А.В. Елецкий. УФН 172, 4, 401 (2002).

[4] J. Di, S. Fang, F.A. Moura, D.S. Galvao, J. Bykova, A. Aliev, M.J.d. Andrade, X. Lepro, N. Li, C. Haines, R. Ovalle-Robles, D. Qian, R.H. Baughman. Adv. Mater. 28, 6598 (2016).

[5] Y. Bai, R. Zhang, X. Ye, Z. Zhu, H. Xie, B. Shen, D. Cai, B. Liu, C. Zhang, Z. Jia, S. Zhang, X. Li, F. Wei. Nature Nanotechnol. 13, 589 (2018).

[6] B.C. Liu, T.J. Lee, S.H. Lee, C.Y. Park, C.J. Lee. Chem. Phys. Lett. 377, 55 (2003).

[7] Y. Li, X. Zhang, X. Tao, J. Xu, W. Huang, J. Luo, Z. Luo, T. Li, F. Liu, Y. Bao, H.J. Geise. Carbon 43, 2, 295 (2005).

[8] Э.Г. Раков. Успехи химии 82, 1, 27 (2013).

[9] T. Hertel, R.E. Walkup, P. Avouris. Phys. Rev. B 58, 20, 13870 (1998).

[10] J. Xie, Q. Xue, H. Chen, D. Xia, C. Lv, M. Ma. J. Phys. Chem. C 114, 2100 (2010).

[11] X. Yuan, Y. Wang. Nanotechnol. 29, 075705 (2018).

[12] A.V. Savin, E.A. Korznikova, S.V. Dmitriev. Phys. Rev. B 92, 035412 (2015).

[13] А.В. Савин, Е.А. Корзникова, С.В. Дмитриев. ФТТ 57, 11, 2278 (2015).

[14] А.В. Савин, О.И. Савина. ФТТ 61, 11, 2257 (2019).

Редактор Т.Н. Василевскал 